\title{
Health Care Investment: The Case of Multiple Sources of Risk
}

\author{
Octave Jokung ${ }^{1} \cdot$ Sovan Mitra ${ }^{2} \oplus$
}

Published online: 8 November 2019

(c) The Author(s) 2019

\begin{abstract}
This paper analyses the effect of a bivariate risk on the optimal expenses in health care and gives conditions under which any change in the bivariate risk with respect to the $\left(s_{1}, s_{2}\right)$-increasing concave order decreases the expenses in health care. Increasing risk increases the demand for health care for risk-averse and prudent individuals in the multivariate sense. Positive (negative) dependence increases (decreases) expenses in health care. Increasing the correlation produces the same results. Furthermore, the uncertainty surrounding the effectiveness of medical treatments amplifies the effect of any change in wealth and health risks. We also present some policy implications.
\end{abstract}

Keywords Dependence $\cdot$ Health uncertainty $\cdot$ Health care $\cdot$ Pair-wise risk aversion . Increase in risk $\cdot$ Stochastic dominance

JEL Classification D81 · G11 · I11

\section{Introduction}

Uncertainty affects the demand for health care (see for instance Grossman 1972; Cropper 1977; Dardanoni and Wagstaff 1990; Finkelstein et al. 2019; Ahangar et al. 2018). Three sources of uncertainty are present in the literature: wealth risk (that is the potential loss of wealth), health risk (that is the potential reduction in one's health) and the risk of the effectiveness of medical care (that is the potential ineffectiveness of medical care). Dardanoni and Wagstaff (1990) give conditions under which uncertainty surrounding health care effectiveness increases medical care. These conditions are expressed with partial derivatives and cross derivatives of the

Sovan Mitra

sovan.mitra@liverpool.ac.uk

1 Institut d'Administration des Entreprises, Universite Polytechnique Hauts-de-France, Valenciennes, France

2 Department of Mathematics, University of Liverpool, Liverpool, UK 
bivariate utility function of consumption and health and the cross derivatives play an important role (see Eeckhoudt et al. 2007).

Palumbo (1999) shows that uncertain health expenses generate precautionary savings. Pang and Warshawsky (2010) demonstrate that uninsured health expenses lower non-health consumption at all ages and that the wealth available for consumption after deduction of health expenses becomes more volatile in the presence of additional background risk. The uncertainty in health expenses implies precautionary savings and shifts from risky assets to risk-free assets. Edwards (2008) presents a theoretical model in which portfolio shares are based on health risk. Edwards (2008) shows that health status affects portfolio choice through different channels. Heaton and Lucas (2000) investigate whether risky human capital affects the demand for financial assets. Health status influences the level of risk aversion which in turn affects the portfolio allocation.

Rosen and $\mathrm{Wu}$ (2004) show that poor health increases the proportion of financial wealth held in safe assets and decreases the proportion held in other asset categories. Fan and Zhao (2009) show that adverse health shocks motivate a safer portfolio choice. That is, health shocks shift investment from risky assets towards less risky assets. Meer et al. (2003) analyse the impact of wealth on health status and show that wealth-health connection is not driven by short run changes in wealth. However, changes in wealth have a strong correlation with changes in health. All the papers highlight the relationship between health status and portfolio selection generally with empirical studies. By considering saving decisions and health investment, Denuit et al. (2009) show that the optimal decision variable decreases as the pair of risks increases, according to bivariate increasing concave dominance rules. To the best of our knowledge, these papers lack important features because they have examined only the action of health risk on wealth risk but not the way wealth risk acts on health risk.

In our approach, wealth risk can play the role of a background risk. Furthermore we analyse the effect of the two risks taking together on the demand for health care. According to the literature (Keenan et al. 2008; Gollier and Pratt 1996; Kimball 1993; Pratt and Zeckhauser 1987, among others), when facing background risks, the decision-makers should bear less endogenous risk. In our framework, the decision-makers should increase their demand for health care. We put emphasis on the simultaneous effects wealth and health risks have on the demand for health care. We investigate the effect of wealth risk on the willingness to bear health risk.

Our objective is fourfold. Firstly, we aim to analyse the effect of risky wealth on the optimal health care investment with a risky health status. This will shed some the light on the interaction between health investment and wealth situation (riskless or risky). Secondly, we aim to analyse the effect of an increase in both wealth and health risk on the demand for health care. Thirdly, we study the effect of the dependence on the health care demand between the two sources of risk. Fourth, we analyse how the effect of uncertainty surrounding the effectiveness of medical treatments affects medical care expenses.

The paper is organised as follows. Section 2 presents the general model. In Sect. 3 we analyse how an increase in the pair of risks, according to the bivariate increasing concave dominance, affects health care investment. Section 4 presents 
the modification of the health care demand due to the introduction of a second source of risk when facing one source of risk. Section 5 analyses the effect of an increase in one source of risk on the demand for health care. The next section deals with the dependence between the two sources of risks. In Sect. 7 we analyse the demand for medical care is affected by uncertainty surrounding the effectiveness of medical treatments. We finally end the paper with a conclusion.

\section{The General Model}

We assume that the decision-maker faces a one-period model such that at time zero, he has an exogenous wealth $y_{0}$, he knows his current health state $h_{0}$ and chooses the expenses in health care, $m_{X, H}$, to maximise his expected utility given by:

$$
u\left(y_{0}-m_{X, H}, h_{0}\right)+E u\left(X, \psi\left(m_{X, H}, H\right)\right)
$$

We assume that the level of health, taking into account the investment in health care at the end of the period, is given by $\psi(m, H)$ with at least $\frac{\partial \psi}{\partial H} \geq 0, \frac{\partial \psi}{\partial m} \geq 0, \frac{\partial^{2} \psi}{\partial H^{2}} \leq 0$ and $\frac{\partial^{2} \psi}{\partial m^{2}} \leq 0$ as in Dardanoni and Wagstaff (1990), with $\psi(0, h)=h$. For $\psi$ gives the amount of health produced by medical care and elements such as nutritional food with input $m$ in state $H$. $X$ denotes the final risky wealth whereas $H$ stands for the future risky health status. This level of health is risky because it is affected by changes in the climate, exposure to viruses and so on. $X$ can be viewed as a risky portfolio (assets, savings, etc.) or a risky income.

In all what follows $f^{\left(\mathrm{k}_{1}, \mathrm{k}_{2}\right)}$ stands for the $\left(k_{1}, k_{2}\right)$ th partial derivative of the bivariate function $f$.

The utility function is at least such that:

$$
\begin{gathered}
u^{(1,0)}(c, h)>0, u^{(0,1)}(c, h)>0, u^{(2,0)}(c, h)<0, u^{(0,2)}(c, h)<0, \\
u^{(0,2)}(c, h) u^{(2,0)}(c, h)>\left[u^{(1,1)}(c, h)\right]^{2} .
\end{gathered}
$$

The first-order condition is given by:

$$
-u^{(1,0)}\left(y_{0}-m, h_{0}\right)+E\left[\psi^{(1,0)}(m, H) u^{(0,1)}(X, \psi(m, H))\right]=0 .
$$

The second-order condition is given by:

$$
\begin{aligned}
& u^{(2,0)}\left(y_{0}-m, h_{0}\right)+E\left[\psi^{(2,0)}(m, H) u^{(0,1)}(X, \psi(m, H))\right] \\
& +E\left[\left[\psi^{(1,0)}(m, H)\right]^{2} u^{(0,2)}(X, \psi(m, H))\right] \leq 0 .
\end{aligned}
$$

This condition is fulfilled thanks to our hypothesis.

In what follows, we consider two general cases concerning the health production function: an additive form $\psi(m, H)=H+\Phi(m)$ like Selden (1993) and a 
multiplicative form $(m, H)=H(1+\varphi(m))$ like Dardanoni and Wagstaff (1987). This formulation encompasses that of Chang (1996).

\section{Changes in Health and Wealth Risks}

We now introduce bivariate stochastic dominance and our main results.

\subsection{Bivariate Stochastic Dominance}

We use the concept of bivariate increasing concave dominance, to analyse how health care investment is affected by a change in the pair of risks $(X, H)$. We note in passing that univariate analysis is a popular paradigm for decision analysis as well as risk analysis. However we utilise bivariate analysis to explicitly understand the relationship between random variables.

As Denuit et al. (1999b) have done, let us define the class of $\left(s_{1}, s_{2}\right)$-increasing concave functions, denoted by $\mathcal{U}_{s_{1}, s_{2}-i c v}$ as follows:

Definition $1 u \in \mathcal{U}_{s_{1}, s_{2-i c v}}$ if and only if

$$
(-1)^{k_{1}+k_{2}+1} u^{\left(k_{1}, k_{2}\right)}(c, h) \geq 0 \quad \forall k_{1}=0, \ldots, s_{1} \forall k_{2}=0, \ldots, s_{2} ; k_{1}+k_{2} \geq 1
$$

With $u^{\left(k_{1}, k_{2}\right)}(c, h)=\frac{\partial^{k_{1}+k_{2}} u(c, h)}{\partial c^{k_{1}} \partial h^{k_{2}}}$ the $\left(k_{1}, k_{2}\right)$ th partial derivative of the bivariate utility function $u$.

We assume that all the partial and the cross derivatives exist up to order $s_{1}$ and $s_{2}$ for the first and second attributes respectively. The common preferences of all the decision-makers with $\left(s_{1}, s_{2}\right)$-increasing concave utility functions generate the $\left(s_{1}, s_{2}\right)$-increasing concave dominance rule, named the $\left(s_{1}, s_{2}\right)$-increasing concave order. Denuit et al. (1999b) give the following result concerning bivariate random variables:

Assume that $\left(X_{1}, H_{1}\right)$ is dominated by $\left(X_{2}, H_{2}\right)$ in the $\left(\mathrm{s}_{1}, \mathrm{~s}_{2}\right)$-increasing concave order, denoted by $\left(X_{1}, H_{1}\right) \preccurlyeq_{\mathrm{s}_{1}, \mathrm{~s}_{2}-\mathrm{icv}}\left(X_{2}, H_{2}\right)$, then for all functions $u$ in $\mathcal{U}_{\mathrm{s}_{1}, \mathrm{~s}_{2-\text { icv }}}$, we have $\mathrm{E} u\left(X_{1}, H_{1}\right) \leq \mathrm{E} u\left(X_{2}, H_{2}\right)$. In fact $\left(X_{1}, H_{1}\right)$ represents an $\left(s_{1}, s_{2}\right)$ -increase in risk of $\left(X_{2}, H_{2}\right)$.

We note in passing that alternative decision making and economic models exist in addition to stochastic dominance. Consequently, alternative models and decision making methods may result in alternative conclusions to the ones presented in the paper, in particular the behavioural economics models may lead to significantly different results. However, stochastic dominance is a frequently employed decision making model in economics and with respect to the literature in this area, hence we apply this method to our paper. We also note in passing that alternative risk analysis and management methods exist to those discussed in the paper [for instance Markowitz portfolio theory and risk management (see Markowitz (1952)]. Whilst we acknowledge their importance to the literature, the focus on 
this paper is on risk analysis and management in the context of a decision-maker with facing simultaneous and bivariate risk (namely health and wealth risk).

\subsection{The Main Results}

We want to point out the effect of the change in the pair of risks (health and wealth risks) on the demand for health care. To this end, we compare the optimal decision with two different vectors, namely $\left(X_{1}, H_{1}\right)$ and $\left(X_{2}, H_{2}\right)$ such that the latter dominates the former according to the $\left(s_{1}, s_{2}\right)$-increasing concave dominance rule. Let us denote by $m_{X_{1}, H_{1}}^{*}\left(m_{X_{2}, H_{2}}^{*}\right.$, respectively) the optimal decision when the bivariate risk is given by $\left(X_{1}, H_{1}\right)\left[\left(X_{2}, H_{2}\right)\right.$ respectively]. We give conditions under which improving the bivariate risk leads to a decrease of the optimal investment in health care. We consider the case of multiplicative health production function and that of additive health production function.

\subsubsection{The Case of a Multiplicative Health Production Function}

Firstly, let us consider the case of multiplicative health production function. The health production function takes the following form:

$$
\psi(m, H)=H+H \varphi(m)
$$

with

$$
\varphi(m) \geq 0, \varphi^{\prime}(m) \geq 0, \varphi^{\prime \prime}(m) \leq 0 \text { and } \varphi(0)=0
$$

We have the following result:

Theorem 1 Assume the health production function is multiplicative. If $u \in \mathcal{U}_{s_{1}, s_{2-i c v}}$ and

$$
\forall c, h \quad-h \frac{u^{\left(k_{1}, k_{2}+1\right)}(c, h)}{u^{\left(k_{1}, k_{2}\right)}(c, h)} \geq k_{2} \quad \forall k_{1}=0, \ldots, s_{1} \quad \forall k_{2}=0, \ldots, s_{2} ; \quad k_{1}+k_{2} \geq 1
$$

then: $\left(X_{1}, H_{1}\right) \preccurlyeq_{s_{1}, s_{2-i c v}}\left(X_{2}, H_{2}\right) \Rightarrow m_{X_{1}, H_{1}}^{*} \geq m_{X_{2}, H_{2}}^{*}$.

Alternatively, if $u \in \mathcal{U}_{s_{1}, s_{2-i c v}}$ and

$$
-h \frac{u^{\left(k_{1}, k_{2}+1\right)}(c, h)}{u^{\left(k_{1}, k_{2}\right)}(c, h)} \geq k_{2} \quad \forall k_{1}=0, \ldots, s_{1} \quad \forall k_{2}=0, \ldots, s_{2} ; \quad k_{1}+k_{2} \geq 1
$$

then: $\left(X_{1}, H_{1}\right) \preccurlyeq_{s_{1}, s_{2-i c v}}\left(X_{2}, H_{2}\right) \Rightarrow m_{1}^{*} \geq m_{2}^{*}$.

Proof $m_{2}^{*} \leq m_{1}^{*}$ if and only if the first-order condition with $\left(X_{2}, H_{2}\right)$ expressed at $m_{1}^{*}$ is negative. That is:

$$
-u^{(1,0)}\left(y_{0}-m_{1}^{*}, h_{0}\right)+E\left[\varphi^{\prime}\left(m_{1}^{*}\right) H_{2} u^{(0,1)}\left(X_{2}, H_{2}\left(1+\varphi\left(m_{1}^{*}\right)\right)\right)\right] \leq 0
$$




$$
\begin{gathered}
\Leftrightarrow \varphi^{\prime}\left(m_{1}^{*}\right) E\left[H_{2} u^{(0,1)}\left(X_{2}, H_{2}\left(1+\varphi\left(m_{1}^{*}\right)\right)\right)\right] \leq u^{(1,0)}\left(y_{0}-m_{1}^{*}, h_{0}\right) \\
\Leftrightarrow E\left[H_{2} u^{(0,1)}\left(X_{2}, H_{2}\left(1+\varphi\left(m_{1}^{*}\right)\right)\right)\right] \leq E\left[H_{1} u^{(0,1)}\left(X_{1}, H_{1}\left(1+\varphi\left(m_{1}^{*}\right)\right)\right)\right]
\end{gathered}
$$

due to the first-order condition concerning $m_{1}^{*}$. This condition holds if and only if

$$
\begin{gathered}
-h u^{(0,1)}\left(c, h+h \varphi\left(m_{1}^{*}\right)\right) \in \mathcal{U}_{s_{1}, s_{2-i c v}} \\
\Leftrightarrow v(c, h)=-h u^{(0,1)}(c, h) \in \mathcal{U}_{s_{1}, S_{2-i c v}}
\end{gathered}
$$

or equivalently if and only if:

$$
(-1)^{k_{1}+k_{2}+1} v^{\left(k_{1}, k_{2}\right)}(c, h) \geq 0 \quad \forall c, h, \quad \forall k_{1}=0, \ldots, s_{1}, \quad \forall k_{2}=0, \ldots, s_{2} ; \quad k_{1}+k_{2} \geq 1
$$

Remark that

$$
\begin{aligned}
v^{\left(k_{1}, k_{2}\right)}(c, h)= & -k_{2}\left(1+\varphi\left(m_{1}^{*}\right)\right)^{k_{2}-1} u^{\left(k_{1}, k_{2}\right)}\left(c, h+h \varphi\left(m_{1}^{*}\right)\right) \\
& -h\left(1+\varphi\left(m_{1}^{*}\right)\right)^{k_{2}} u^{\left(k_{1}, k_{2}+1\right)}\left(c, h+h \varphi\left(m_{1}^{*}\right)\right) .
\end{aligned}
$$

Therefore, the condition to fulfil becomes:

$$
\begin{gathered}
(-1)^{k_{1}+k_{2}+1}\left[-k_{2}\left(1+\varphi\left(m_{1}^{*}\right)\right)^{k_{2}-1} u^{\left(k_{1}, k_{2}\right)}\left(c, h+h \varphi\left(m_{1}^{*}\right)\right)-h\left(1+\varphi\left(m_{1}^{*}\right)\right)^{k_{2}} u^{\left(k_{1}, k_{2}+1\right)}\right. \\
\left.\left(c, h+h \varphi\left(m_{1}^{*}\right)\right)\right] \geq 0, \quad \forall k_{1}=0, \ldots, s_{1}, \quad \forall k_{2}=0, \ldots, s_{2} ; \quad k_{1}+k_{2} \geq 1
\end{gathered}
$$

Equivalently:

$$
\begin{aligned}
& (-1)^{k_{1}+k_{2}+1} u^{\left(k_{1}, k_{2}\right)}\left(c, h+h \varphi\left(m_{1}^{*}\right)\right)\left(1+\varphi\left(m_{1}^{*}\right)\right)^{k_{2}-1} \\
& \quad\left[-k_{2}-h\left(1+\varphi\left(m_{1}^{*}\right)\right) \frac{u^{\left(k_{1}, k_{2}+1\right)}\left(c, h+h \varphi\left(m_{1}^{*}\right)\right)}{u^{\left(k_{1}, k_{2}\right)}\left(c, h+h \varphi\left(m_{1}^{*}\right)\right)}\right] \geq 0, \forall k_{1}=0, \ldots, s_{1}, \\
& \quad \forall k_{2}=0, \ldots, s_{2} ; \quad k_{1}+k_{2} \geq 1
\end{aligned}
$$

The term before the brackets is positive and the condition holds if:

$$
\begin{gathered}
-h\left(1+\varphi\left(m_{1}^{*}\right)\right) \frac{u^{\left(k_{1}, k_{2}+1\right)}\left(c, h+h \varphi\left(m_{1}^{*}\right)\right)}{u^{\left(k_{1}, k_{2}\right)}\left(c, h+h \varphi\left(m_{1}^{*}\right)\right)} \geq k_{2} \\
\forall k_{1}=0, \ldots, s_{1}, \quad \forall k_{2}=0, \ldots, s_{2} ; \quad k_{1}+k_{2} \geq 1
\end{gathered}
$$


The condition involves the generalised coefficients of relative risk aversion in health defined as follows:

$$
R R_{k_{1}, k_{2}}(c, h)=-h \frac{u^{\left(k_{1}, k_{2}+1\right)}}{u^{\left(k_{1}, k_{2}\right)}}(c, h) .
$$

Also the condition states that this generalised coefficient must be greater than a benchmark value given by $k_{2}$.

Recall that in the univariate framework the coefficient of relative risk aversion is given by $-h \frac{v^{\prime \prime}(h)}{v^{\prime}(h)}$, whereas the coefficient of relative risk aversion of order $k_{2}$ corresponds to $-h \frac{v^{\left(k_{2}\right)}(h)}{v^{\left(k_{2}-1\right)}(h)}$ where $v$ is the univariate utility function of health. In fact, $R R_{k_{1}, k_{2}}(c, h)$ corresponds to the coefficient of relative risk aversion of order $k_{2}$ of the utility function $u^{\left(k_{1}, 0\right)}$ of order $k_{2}$, with respect to the second attribute. Risk aversion of order two corresponds to usual risk aversion (introduced by Pratt 1964), risk aversion of order three corresponds to prudence (introduced by Kimball 1990), risk aversion of order four corresponds to temperance (introduced by Kimball 1993), risk aversion of order five corresponds to edginess (introduced by Lajeri-Chaherli 2004) and so forth.

Eeckhoudt et al. (2007) show that the respective benchmark values of relative risk aversion and prudence are one and two. In fact, the benchmark value for $-h \frac{v^{\left(k_{2}+1\right)}(h)}{v^{\left(k_{2}\right)}(h)}$

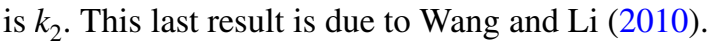

Therefore, the condition stands that for all values of $k_{2}$ up to $s_{2}$ the coefficients of relative risk aversion of $u^{\left(k_{1}, 0\right)}$, with respect to the second attribute (the one concerned by the decision variable) at any order up to $s_{2}$, are less than their respective order.

The use of the multiplicative health production function implies that the conditions in Theorem 1 are expressed in terms of relative risk aversion of higher order (relative risk aversion, relative prudence, relative temperance...). For example, if we consider the special case with $s_{1}=1$ and $s_{2}=1$, the conditions to be fulfilled are the following: $-h \frac{u^{(0,2)}}{u^{(0,1)}}(c, h) \geq 1 \forall c, h$ : Relative risk aversion of $u^{(0,0)}=u$ with respect to health greater than one. $-h \frac{u^{(1,2)}}{u^{(1,1)}}(c, h) \geq 1 \forall c, h$ : Relative risk aversion of $u^{(1,0)}$ with respect to health greater than one. $u^{(1,1)}(c, h) \leq 0 \forall c, h$ : Pair-wise risk aversion (multivariate risk aversion) and the utility function is increasing with the two attributes.

The condition $u^{(1,1)}(c, h)=\frac{\partial^{2} u}{\partial c \partial h} \leq 0$ corresponds to multivariate risk aversion introduced by Richard (1975), and named pair-wise risk aversion by Scarsini (1985), as follows:

Definition 2 An individual is said to behave in a multivariate risk averse manner if he prefers the lottery $L\left[\left(c_{1}, h_{2}\right),\left(c_{2}, h_{1}\right) ; \frac{1}{2}, \frac{1}{2}\right]$ to the lottery $L\left[\left(c_{1}, h_{1}\right),\left(c, h_{2}\right) ; \frac{1}{2}, \frac{1}{2}\right]$ with $c_{1} \leq c_{2}$ and $h_{1} \leq h_{2}$.

In the expected utility framework, this definition is equivalent to: 


$$
u\left(c_{1}, h_{2}\right)+u\left(c_{2}, h_{1}\right) \geq u\left(c_{1}, h_{1}\right)+u\left(c_{2}, h_{2}\right) \forall c_{1} \leq c_{2}, \quad h_{1} \leq h_{2} .
$$

That is, the utility function is sub-modular or 2-antitone. According to Richard (1975), "The decision-maker prefers getting some of the 'best' and some of the 'worst' to taking a chance on all [of] the 'best' or all [of] the 'worst'". Or equivalently, the decision-maker prefers having low wealth with good health or high wealth with poor health instead of having high wealth with good health or low wealth with poor health. $u^{(1,1)} \leq 0$ means that the marginal utility of wealth decreases with health status and vice versa.

\subsubsection{The Case of Additive Health Production Function}

Second, let us consider the case of additive production function. The health production function takes the following form:

$$
\psi(m, H)=H+\Phi(m),
$$

with at least

$$
\Phi(m) \geq 0, \Phi^{\prime}(m) \geq 0, \Phi^{\prime \prime}(m) \leq 0 \text { and } \Phi(0)=0 .
$$

Recall that the functional form chosen by Denuit, Eeckhoudt and Menegatti (2009) was the following:

$$
\psi(m, H)=H+m \text {. }
$$

The decision maker chooses the amount $m$ invested in health care to maximise his expected utility:

$$
u\left(y_{0}-m, h_{0}\right)+E u(X, H+\Phi(m)) .
$$

The first-order condition is given by:

$$
-u^{(1,0)}\left(y_{0}-m, h_{0}\right)+\Phi^{\prime}(m) E\left[u^{(0,1)}(X, H+\Phi(m))\right]=0 .
$$

The second-order condition is satisfied. Thus, the optimum is an interior optimum.

The following result which concerns the additive production function generalises the result from Denuit et al. (2009):

Theorem 2 Assume the health production function is additive. If $u \in \mathcal{U}_{s_{1}, s_{2+1-i c v}}$ then $\left(X_{1}, H_{1}\right) \preccurlyeq_{s_{1}, s_{2-i c v}}\left(X_{2}, H_{2}\right) \Rightarrow m_{X_{1}, H_{1}}^{*} \geq m_{X_{2}, H_{2}}^{*}$. Alternatively, if $u \in \mathcal{U}_{s_{1}, s_{2+1-i c v}}$ then $\left(X_{1}, H_{1}\right) \preccurlyeq_{s_{1}, s_{2-i c v}}\left(X_{2}, H_{2}\right) \Rightarrow m_{1}^{*} \geq m_{2}^{*}$.

Proof $m_{2}^{*} \leq m_{1}^{*}$ if and only if the first-order condition with $\left(X_{2}, H_{2}\right)$ expressed at $m_{1}^{*}$ is negative. That is:

$$
\begin{gathered}
-u^{(1,0)}\left(y_{0}-m_{1}^{*}, h_{0}\right)+E\left[\Phi^{\prime}\left(m_{1}^{*}\right) u^{(0,1)}\left(X_{2}, H_{2}+\Phi\left(m_{1}^{*}\right)\right)\right] \leq 0 \\
\Leftrightarrow E\left[u^{(0,1)}\left(X_{2}, H_{2}+\Phi\left(m_{1}^{*}\right)\right)\right] \leq E\left[u^{(0,1)}\left(X_{1}, H_{1}+\Phi\left(m_{1}^{*}\right)\right)\right]
\end{gathered}
$$

This condition holds if and only if 


$$
\begin{aligned}
& -u^{(0,1)}\left(c, h+\Phi\left(m_{1}^{*}\right)\right) \in \mathcal{U}_{s_{1}, s_{2-i c v}} \\
& \Leftrightarrow v(c, h)=-u^{(0,1)}(c, h) \in \mathcal{U}_{s_{1}, s_{2-i c v}}
\end{aligned}
$$

or equivalently if and only if:

$$
\begin{aligned}
& (-1)^{k_{1}+k_{2}+1} v^{\left(k_{1}, k_{2}\right)}(c, h) \geq 0 \quad \forall c, h, \quad \forall k_{1}=0, \ldots, s_{1}, \forall k_{2}=0, \ldots, s_{2} ; \quad k_{1}+k_{2} \geq 1 \\
& \Leftrightarrow(-1)^{k_{1}+\left(k_{2}+1\right)+1} u^{\left(k_{1}, k_{2}+1\right)}(c, h) \geq 0 \quad \forall c, h, \quad \forall k_{1}=0, \ldots, s_{1}, \forall k_{2}=0, \ldots, s_{2} ; \quad k_{1}+k_{2} \geq 1
\end{aligned}
$$

The condition is fulfilled when $u \in \mathcal{U}_{s_{1}, s_{2+1-i c v}}$.

For example, if we consider the particular case with $s_{1}=1$ and $s_{2}=1$, the conditions to be fulfilled in order to see a decrease in the demand for health care after an increase in the pair of risks according to the $(1,1)$ - increasing concave order are the following:

$u^{(0,2)} \leq 0$ : Risk aversion with respect to health;

$u^{(1,1)} \leq 0$ : Pair-wise risk aversion;

$u^{(1,2)} \geq 0$ : Cross-prudence with respect to health;

and the utility function increases with the two attributes as usual.

The condition $u^{(1,2)} \geq 0$ corresponds to cross-prudence introduced by Eeckhoudt et al. (2007) as follows:

Definition 3 Cross-prudence with respect to the second attribute of the utility function is equivalent to the preference for the 50-50 lottery $\left[\left(c_{1}, h_{2}\right),\left(c_{2}+\tilde{\mathcal{E}}_{2}, h_{2}\right)\right]$ over the 50-50 lottery $\left[\left(c_{1}+\tilde{\mathcal{E}}_{2}, h_{2}\right),\left(c_{2}, h_{2}\right)\right]$ with $c_{1} \leq c_{2}, h_{2}$, and $\widetilde{\mathcal{E}}_{2}$ such that $E\left(\tilde{\mathcal{E}}_{2}\right)=0$.

Jokung (2011) related cross-prudence to risk apportionment of order $(1,2)$. Risk apportionment means that the individual prefers to disaggregate risks across the different states of nature. In addition, it is supposed that the individual prefers to get some of the 'good' and some of the 'bad', rather than taking a chance on all of the 'good' and all of the 'bad' (pair-wise risk aversion).

\section{On One Risk to Two Risks}

We start with the situation without uncertainty and we tackle two cases: first the decision-maker faces a health risk and we introduce a wealth risk; second, the decision-maker deals with a wealth risk and we add a health risk. We want to analyse the effect of the introduction of the risk on the demand for health care. 
Let $m_{E(X), H}^{*}, m_{X, E(H)}^{*}$ and $m_{E(X), E(H)}^{*}$ denote the decisions with the riskless wealth, with the riskless health and with both riskless wealth and riskless health, respectively.

\subsection{Health Risk}

In the initial situation, without any risk, the decision-maker maximises his utility and he determines the optimal expenses in medical care without uncertainty. First, we introduce the health risk (we replace the mean of the random health status by the random health status itself), the decision-maker chooses the expenses in health care when facing $(E(X), H)$ and the optimal expenses in health care are $m_{E(X), H}^{*}$. The convexity of the marginal utility with respect to health is sufficient to guarantee an increase in the demand for health care. We are in presence of a precautionary behaviour. The decision-maker increases the amount invested in health care in order to take into account the introduction of the uncertainty. This result is analogous to that of Palumbo (1999) concerning precautionary savings. The convexity of the marginal utility function plays an important role. Thus $m_{E(X), H}^{*} \geq m_{E(X), E(H)}^{*}$ when $u^{(0,3)} \geq 0$ (prudence) with an additive health production function. The condition becomes $-h \frac{u^{(0,3)}}{u^{(0,2)}} \geq 2$ (relative prudence greater than two) with a multiplicative health production function.

Now, our aim is to compare $m_{E(X), H}^{*}$ with $m_{X, H}^{*}$, obtained when facing $(X, H)$. Intuitively, this particular increase in wealth risk (for example changing a safe portfolio for a risky one) will have an equivalent effect as an increase in income uncertainty. In the univariate framework, as shown by Kimball and Weil (2009) decreasing absolute risk aversion is sufficient to ensure a prudent behaviour, and consequently an increase in the demand for health care. We generalise this result.

Proposition 1 Assume the health production function is multiplicative.

1. If $-h \frac{u^{(0,3)}}{u^{(0,2)}}(c, h) \geq 2$, then $m_{E(X), H}^{*} \geq m_{E(X), E(H)}^{*}$.

2. If $u \in \mathcal{U}_{2,1,-i c v}$ and $-h \frac{u^{(0,2)}}{u^{(0,1)}}(c, h) \geq 1 ;-h \frac{u^{(1,2)}}{u^{(1,1)}}(c, h) \geq 1 ;-h \frac{u^{(2,2)}}{u^{(2,1)}}(c, h) \geq 1 \forall c, h$ then $m_{X, H}^{*} \geq m_{E(X), H^{*}}^{*}$

Proof The first-order condition expressed at $m=m_{E(X), E(H)}^{*}$ is non-negative if and only if:

$$
\begin{gathered}
-u^{(1,0)}\left(y_{0}-m, h_{0}\right)+\varphi^{\prime}(m) E H u^{(0,1)}(E(X), H+\varphi(m) H) \geq 0 \\
\Leftrightarrow E H u^{(0,1)}(E(X), H+\varphi(m) H) \geq E(H) u^{(0,1)}(E(X), E(H)+\varphi(m) E(H))
\end{gathered} .
$$

This is true if $h u^{(0,1)}$ is a convex function of health. That is,

$$
2 u^{(0,2)}(c, h)+h u^{(0,3)}(c, h) \geq 0 .
$$

Thus the result follows. 
Concerning the second part of the proposition, remark that $(X, H) \preccurlyeq_{2,1_{-i c v}}(E(X), H)$ and apply Theorem 1.

The conditions to guarantee that $m_{X, H}^{*} \geq m_{E(X), H}^{*}$ imply that the coefficients of relative risk aversion of $u^{(0,0)}, u^{(1,0)}$ and $u^{(2,0)}$ to be greater than one but also the utility function to exhibit risk aversion $\left(u^{(0,2)} \leq 0\right.$ and $\left.u^{(2,0)} \leq 0\right)$, pair-wise risk aversion $\left(u^{(1,1)} \leq 0\right)$, cross-prudence $\left(u^{(1,2)} \geq 0\right.$ and $\left.u^{(2,1)} \geq 0\right)$, temperance $\left(u^{(2,2)} \leq 0\right)$.

Proposition 1 bis Assume the health production function is additive.

1. If $u^{(0,3)} \geq 0$, then $m_{E(X), H}^{*} \geq m_{E(X), E(H)}^{*}$.

2. If $u \in \mathcal{U}_{2,2,-i c v}$ then $m_{X, H}^{*} \geq m_{E(X), H}^{*}$.

Proof The first-order condition with the health risk expressed at $m=m_{E(X), E(H)}^{*}$ is non-negative if and only if:

$$
\begin{aligned}
& -u^{(1,0)}\left(y_{0}-m, h_{0}\right)+\Phi^{\prime}(m) E u^{(0,1)}(E(X), H+\Phi(m)) \geq 0 \\
& \Leftrightarrow E u^{(0,1)}(E(X), H+\Phi(m)) \geq u^{(0,1)}(E(X), E(H)+\Phi(m))
\end{aligned}
$$

This is true if $u^{(0,1)}$ is convex with respect to health. That is $u^{(0,3)} \geq 0$. For the second part of the proposition, remark that $(X, H) \preccurlyeq_{, 12_{-i c v}}(X, E(H))$ and apply Theorem 2.

The details concerning the conditions to be fulfilled in order to ensure that the introduction of the wealth risk in presence of the health risk will increase the demand for medical care are:

- Increase in both attributes;

- Concavity of the utility function with respect to both attributes;

- Pair-wise risk aversion;

- Cross-prudence with respect to both arguments;

- Temperance.

The concept of temperance was introduced by Eeckhoudt et al. (2007) as follows:

Definition 4 Temperance is equivalent to the preference for the 50-50 lottery $\left[\left(c+\tilde{\mathcal{E}}_{1}, h\right),\left(c, h+\tilde{\mathcal{E}}_{2}\right)\right]$ over the 50-50 lottery $\left[\left(c+\tilde{\mathcal{E}}_{1}, h+\tilde{\mathcal{E}}_{2}\right),(c, h)\right] \forall c, h$, and $\tilde{\mathcal{E}}_{1}, \tilde{\mathcal{E}}_{2}$ two independent zero-mean random variables.

Temperance corresponds to bivariate risk apportionment of order $(2,2)$. That is, a type of preference for disaggregation of the addition of two independent zero-mean random variables in each attribute of the utility function. 


\subsection{Wealth Risk}

In this section we analyse the effect of the introduction of wealth risk on the demand for health care. We tackle this analysis sequentially, first dealing with the case where there is no uncertainty about health and introduction wealth risk. We then move on to the case where there is uncertainty about health, before introducing wealth risk. In the first case, where there is no uncertainty and we introduce wealth risk, the decision-maker chooses the expenses in health care to maximise expected utility when facing $(X, E(H))$. The optimal expenses in health care are $m_{X, E(H)}^{*}$. These optimal expenses are greater than those without wealth risk if the marginal utility related to health is convex with respect to wealth in case of additive production function. This is precautionary due to saving motives (see Kimball 1990; Drèze and Modigliani 1972; Sandmo 1970; Leland 1968) via the demand for health.

Our aim is to compare $m_{X, E(H)}^{*}$ with $m_{X, H}^{*}$. The decision-maker faces a wealth risk and we introduce a health risk. Therefore, the demand for health care must take into account the increase in global uncertainty. Our intuition is to see an increase in the demand for health care. We have the following results:

Proposition 2 Assume the production function is multiplicative.

1. If $u^{(1,2)} \geq 0$ then $m_{X, E(H)}^{*} \geq m_{E(X), E(H)}^{*}$.

2. If $u \in \mathcal{U}_{1,2_{-i c v}}$ and

$$
\begin{gathered}
-h \frac{u^{(0,2)}}{u^{(0,1)}}(c, h) \geq 1 ;-h \frac{u^{(1,2)}}{u^{(1,1)}}(c, h) \geq 1 ;-h \frac{u^{(0,3)}}{u^{(0,2)}}(c, h) \geq 2 ;-h \frac{u^{(1,3)}}{u^{(1,2)}}(c, h) \geq 2 \forall c, h, \text { then } \\
m_{X, H}^{*} \geq m_{X, E(H)}^{*} .
\end{gathered}
$$

Proof The first-order condition expressed at $m=m_{E(X), E(H)}^{*}$ is non-negative if and only if:

$$
\begin{gathered}
-u^{(1,0)}\left(y_{0}-m, h_{0}\right)+\varphi^{\prime(m)} E(H) E u^{(0,1)}(X, E(H)+\varphi(m) E(H)) \geq 0 \\
\Leftrightarrow E u^{(0,1)}(X, E(H)+\varphi(m) E(H)) \geq E u^{(0,1)}(E(X), E(H)+\varphi(m) E(H))
\end{gathered} .
$$

This is true if $u^{(0,1)}$ is convex with respect to wealth. That is, $u^{(1,2)} \geq 0$.

Remark that $(X, H) \preccurlyeq_{1,2_{-i c v}}(X, E(H))$ and apply Theorem 1 .

The conditions to ensure that $m_{X, H}^{*} \geq m_{X, E(H)}^{*}$ are the coefficients of relative risk aversion of $u^{(0,0)}$ and $u^{(1,0)}$ with respect to health to be greater than one and the coefficients of relative prudence of $u^{(0,0)}$ and $u^{(1,0)}$ to be greater than two.

Proposition 2 bis Assume the health production function is additive.

1. If $u^{(2,1)} \geq 0$ then $m_{X, E(H)}^{*} \geq m_{E(X), E(H)}^{*}$.

2. If $u \in \mathcal{U}_{1,3_{-i c v}}$ then $m_{X, H}^{*} \geq m_{X, E(H)}^{*}$. 
Proof The first-order condition expressed at $m=m_{E(X), E(H)}^{*}$ is non-negative if and only if:

$$
\begin{aligned}
& -u^{(1,0)}\left(y_{0}-m, h_{0}\right)+\Phi^{\prime}(m) E u^{(0,1)}(X, E(H)+\Phi(m)) \geq 0 \\
& \Leftrightarrow E u^{(0,1)}(X, E(H)+\Phi(m)) \geq u^{(0,1)}(E(X), E(H)+\Phi(m))
\end{aligned}
$$

This is true if $u^{(0,1)}$ is convex with respect to wealth. That is, $u^{(2,1)} \geq 0$.

Concerning the second part of the proposition, remark that $(X, H) \preccurlyeq_{1,2_{-i c v}}(X, E(H))$ and apply Theorem 2.

The first part of Proposition 2 bis corresponds to the bivariate version of the precautionary expenses in medical care due to a prudent decision-maker. The introduction of a second source of risk induces an increase in the demand for health care. There is an extra positive demand for health care due to income risk in the first case and due to health risk in the second case. There is a form of hedge-health care investment, where the decision-maker uses the health care investment to prevent the increase in global risk, following the introduction of the second source of risk (wealth risk or health risk). With an additive health production function, the effect of the introduction of wealth risk in the absence of health risk will be of larger magnitude for those individuals who are cross-prudent with respect to wealth.

\section{Increasing Health or Wealth Risks}

In this section, we increase one of the two risks with respect to the univariate stochastic dominance rule.

\subsection{Stochastic Dominance}

In the univariate framework, consider two random variables $Z$ and $T$ with respective cumulative distribution functions $F$ and $G$ with bounded supports contained within the interval $[a, b]$. Let

$$
\begin{gathered}
F_{1}(x)=F(\mathrm{x}) \text { and } G_{1}(x)=G(\mathrm{x}), \\
F_{n}(x)=\int_{a}^{x} F_{n-1}(z) d z \text { and } G_{n}(x)=\int_{a}^{x} G_{n-1}(z) d z, \quad \forall n \geq 2 .
\end{gathered}
$$

Definition 5 The distribution $F$, or equivalently the random variable $Z$, dominates the distribution $G$, or equivalently the random variable $T$, in the sense of the $N$ th -order stochastic dominance if and only if:

$$
F_{N}(x) \leq G_{N}(x), \quad \forall x \in[a, b],
$$

And $F_{n}(b) \leq G_{n}(b), \forall n=1, \ldots, N-1$. 
We write $G \preccurlyeq_{N} F$ to denote that $F$ dominates $G$ with respect to the $N$ th- order stochastic dominance.

$N=1, N=2$ and $N=3$ correspond to the first-order stochastic dominance (FSD), second-order stochastic dominance (SSD) and third-order stochastic dominance (TSD).

\subsection{Increasing Health Risk}

We tackle two cases, first the health risk is the unique risk $\left(H_{2}\right)$ faced by the decision-maker. The decision-maker determines the optimal amount, $m_{E(X), H_{2}}^{*}$, to maximise his expected utility before the increase in the health risk. After this increase, he or she determines the optimal amount, $m_{E(X), H_{1}}^{*}$, to maximise his or her expected utility where $H_{2}$ dominates $H_{1}$ according to the $s_{2}^{\text {th }}$-order stochastic dominance.

Second, the decision-maker faces health and wealth risk. The amounts to be compared are $m_{X, H_{2}}^{*}$ and $m_{X, H_{1}}^{*}$. We have the following result establishing conditions on the utility function such that a deterioration of the health risk according to the stochastic dominance leads to an increase in the demand for health care. The first part of the proposition corresponds to the case of one source of risk whereas the second part deals with two sources of risk.

Proposition 3 Assume the health production function is multiplicative.

1. If $\forall c, h,-h \frac{u^{\left(0, k_{2}+1\right)}(c, h)}{u^{\left(0, k_{2}\right)}(c, h)} \geq k_{2}, \forall k_{2}=1, \ldots, s_{2}$

then: $H_{1} \preccurlyeq_{s_{2}} H_{2} \Rightarrow m_{E(X), H_{1}}^{*} \geq m_{E(X), H_{2}}^{*}$.

2. If $\forall c, h,-h \frac{u^{\left(0, k_{2}+1\right)}(c, h)}{u^{\left(0, k_{2}\right)}(c, h)} \geq k_{2}, \quad \forall k_{2}=1, \ldots, s_{2}$ and $-h \frac{u^{\left(1, k_{2}+1\right)}(c, h)}{u^{\left(1, k_{2}\right)}(c, h)} \geq k_{2}, \quad \forall k_{2}=0, \ldots, s_{2}$

then: $H_{1} \preccurlyeq_{S_{2}} H_{2} \Rightarrow m_{X, H_{1}}^{*} \geq m_{X, H_{2}}^{*}$

Proof $H_{1} \preccurlyeq_{S_{2}} H_{2}$ implies that $\left(E(X), H_{1}\right) \preccurlyeq_{0, s_{2}}\left(E(X), H_{2}\right)$ and applying Theorem 1 gives the result of part 1 . Notice that $H_{1} \preccurlyeq_{s_{2}} H_{2}$ implies $\left(X, H_{1}\right) \preccurlyeq_{1, s_{2}}\left(X, H_{2}\right)$ and applying Theorem 1 gives the result of the second part.

In considering a change in the health risk with only one source of risk, from $H_{2}$ to $H_{1}$ it is a pure increase in endogenous risk. Recall that the presence of the health risk generates a precautionary behaviour, increasing health risk will reinforce this behaviour. Thus, the decision-maker has incentives to increase the demand for health care. If we consider the case of two sources of risks, the decision-maker faces a background risk and this background risk increases the willingness to bear health risk. So, if in this case, we increase the endogenous risk, the decision-maker will intuitively react by increasing the demand for health care. We recover the same result when the production function is additive. 
Proposition 3 bis Assume the health production function is additive.

1. If $u \in \mathcal{U}_{0, s_{2}+1_{-i c v}}$ then $H_{1} \preccurlyeq_{s_{2}} H_{2} \Rightarrow m_{E(X), H_{1}}^{*} \geq m_{E(X), H_{2}}^{*}$

2. If $u \in \mathcal{U}_{1, s_{2}+1_{-i c v}}$ then $H_{1} \preccurlyeq_{s_{2}} H_{2} \Rightarrow m_{X, H_{1}}^{*} \geq m_{X, H_{2}}^{*}$

Proof The proof is the same as that of Proposition 3 except that we use Theorem 2 instead of Theorem 1.

The two propositions generalise the results of Eeckhoudt and Schlesinger (2008) concerning the effects of the changes in income and interest risks on the demand for savings. In our model, the demand for health care acts like the demand for savings in their approach.

From a policy perspective, this proposition suggests that policy measures which increase the uncertainty surrounding the average health status will tend to result in an increase in the demand for health care. This result generalises that of Dardanoni and Wagstaff (1987).

\subsection{Increasing Wealth Risk}

First, the decision-maker faces only wealth risk and determines the optimal amount, $m_{X_{2}, E(H)}^{*}$ to maximise his expected utility before the increase in the wealth risk. After the increase in wealth risk, he or she determines the optimal amount, $m_{X_{1}, E(H)}^{*}$ to maximise his or her expected utility, where $X_{2}$ dominates $X_{1}$ according to the $s_{1}$ thorder stochastic dominance. Second, the decision-maker faces health and wealth risk. The amounts to be compared are $m_{X_{2}, H}^{*}$ and $m_{X_{1}, H}^{*}$. The first part of the next proposition corresponds to the case of one source of risk, whereas the second part deals with two sources of risk.

Proposition 4 Assume the health production function is multiplicative.

1. If $\forall c, h,-h \frac{u^{\left(k_{1}, 1\right)}(c, h)}{u^{\left(k_{1}, 0,\right)}(c, h)} \geq 0, \forall k_{1}=1, \ldots, s_{1}$

then: $X_{1} \preccurlyeq_{s_{1}} X_{2} \Rightarrow m_{X_{1}, E(H)}^{*} \geq m_{X_{2}, E(H)}^{*}$.

2. If $\forall c, h,-h \frac{u^{\left(k_{1}, 1\right)}(c, h)}{u^{\left(k_{1}, 0\right)}(c, h)} \geq 0, \quad \forall k_{1}=1, \ldots, s_{12} ;-h \frac{u^{u^{\left(k_{1}, 2\right)}(c, h)}}{u^{\left(k_{1}, 1\right)}(c, h)} \geq 1, \quad \forall k_{1}=0, \ldots, s_{1}$, and $-h \frac{u^{\left(k_{1}, 3\right)}(c, h)}{u^{\left(k_{1}, 2\right)}(c, h)} \geq 2, \quad \forall k_{1}=0, \ldots, s_{1}$,

then: $X_{1} \preccurlyeq_{s_{1}} X_{2} \Rightarrow m_{X_{1, H}}^{*} \geq m_{X_{2}, H}^{*}$.

Proof $X_{1} \preccurlyeq_{s_{1}} X_{2}$ implies that $\left(X_{1}, E(H)\right) \preccurlyeq_{s_{1}, 0}\left(X_{2}, E(H)\right)$ and applying Theorem 1 gives the result of part 1 . Notice that $X_{1} \preccurlyeq_{S_{1}} X_{2}$ implies $\left(X_{1}, H\right) \preccurlyeq_{s_{1}, 1}\left(X_{2}, H\right)$ and applying Theorem 1 gives the result of the second part. 
In the first part of the proposition, wealth risk changes from $X_{2}$ to $X_{1}$. That is a pure increase in background risk (exogenous risk). Therefore, this increase in background risk gives the decision-maker incentives to increase the demand for health care in order to hedge the exogenous risk. The conditions, in the second part of the proposition, involve the relative risk aversion being greater than one and the relative prudence being greater than two.

We have the following result when the health production function is additive.

Proposition 4 bis Assume the health production function is additive.

1. If $u \in \mathcal{U}_{s_{1}, 1_{-i c v}}$ then $X_{1} \preccurlyeq_{s_{1}} X_{2} \Rightarrow m_{X_{1}, E(H)}^{*} \geq m_{X_{2}, E(H)}^{*}$.

2. If $u \in \mathcal{U}_{s_{1}, 2_{-i c v}}$ then $X_{1} \preccurlyeq_{s_{1}} X_{2} \Rightarrow m_{X_{1, H}^{*}}^{*} \geq m_{X_{2}, H^{*}}^{*}$

The proof is the same as that of Proposition 4 except that we use Theorem 2 instead of Theorem 1.

For example, assume that the wealth risk increases according to the second order stochastic dominance. When facing only the wealth risk (the health status is riskless), the decision-maker increases the demand for health care if the bivariate utility function exhibits pair-wise risk aversion, risk aversion with respect to wealth and cross-prudence with respect to wealth. If the health status is risky, the conditions to be fulfilled in order to see an increase in the demand for health care after a second degree deterioration of the wealth risk are risk-aversion with respect to the two attributes of the utility function, pair-wise risk aversion, cross-prudence with respect to wealth and health and temperance.

In periods of crisis, namely after an increase in wealth risk, the individuals will react by increasing their demand for health care. Health care plays a hedging role against the deterioration of wealth risk.

\section{The Case of Dependent Health and Wealth Risks}

So far we have studied the effect of the change in the bivariate risk in the decision process. Now, we want to know whether the dependence of the exogenous risk (wealth risk) and the endogenous risk (health risk) affects the decision process. To do so, we consider the case of Bernoulli-distributed variables and the concept of quadrant dependence.

\subsection{Bernoulli-Distributed Variables: Correlation}

Assume that $X$ and $H$ take two values $x_{1}$ and $x_{2}$ for $X$ with $x_{1} \leq x_{2}$ and $h_{1}$ and $h_{2}$ for $H$ with $h_{1} \leq h_{2}$. $x_{1}$ represents low wealth whereas $x_{2}$ corresponds to high wealth. $h_{1}$ corresponds to poor health status whereas $h_{2}$ represents good health status. The joint distribution of $(X, H)$ is given by: 


$$
\begin{gathered}
P\left(X=x_{1}, H=h_{1}\right)=\rho p q, \\
P\left(X=x_{1}, H=h_{2}\right)=p(1-\rho q), \\
P\left(X=x_{2}, H=h_{1}\right)=q(1-\rho p), \\
P\left(X=x_{2}, H=h_{2}\right)=1-p-q+\rho p q .
\end{gathered}
$$

The marginal distribution of $X$ is given by: $P\left(X=x_{1}\right)=p$ and $P\left(X=x_{2}\right)=1-p$.

The marginal distribution of $H$ is given by: $P\left(H=h_{1}\right)=q$ and $P\left(H=h_{2}\right)=1-q$.

There are three cases depending on the value of the dependence parameter $\rho$ :

$\rho>1: X$ and $H$ are positively correlated;

$\rho=1: X$ and $H$ are independent;

$\rho<1: X$ and $H$ are negatively correlated.

Let $m_{\perp}^{*}$ and $m^{*}$ be the optimal decisions in the case of independence and dependence, respectively. Intuition suggests that a positive correlation will induce an increase in the demand for health care because with positive correlation poor health status is likely to be accompanied by low wealth. Therefore, that gives the decision-maker incentives to hedge against this bad situation (low health and poor health) by increasing the demand for health care. The negative correlation will have the opposite effect and will give incentives to reduce the demand for health care because poor health is likely to be accompanied by high wealth. The exogenous risk acts like a hedge-wealth. We have the following propositions:

Proposition 5 Assume the health production function is multiplicative. If $u \in \mathcal{U}_{1,1-i c v}$ with $-h \frac{u^{(1,2)}(c, h)}{u^{(1,1)}(c, h)} \geq 1, \forall c, h$, then

I. If $X$ and $H$ are positively correlated, then $m^{*} \geq m_{\perp}^{*}$.

II. If $X$ and $H$ are negatively correlated, then $m^{*} \leq m_{\perp}^{*}$.

Proof The first-order condition is given by:

$$
\begin{aligned}
- & u^{(1,0)}\left(y_{0}-m, h_{0}\right)+\varphi^{\prime}(m) \rho p q h_{1} u^{(0,1)}\left(x_{1}, h_{1}+\varphi(m) h_{1}\right)+p(1-\rho q) h_{2} u^{(0,1)} \\
& \left(x_{1}, h_{2}+\varphi(m) h_{2}\right)+q(1-\rho p) h_{1} u^{(0,1)}\left(x_{2}, h_{1}+\varphi(m) h_{1}\right) \\
& +(1-p-q+\rho p q) h_{2} u^{(0,1)}\left(x_{2}, h_{2}+\varphi(m) h_{2}\right)=0 .
\end{aligned}
$$

Equivalently: 


$$
\begin{aligned}
- & u^{(1,0)}\left(y_{0}-m, h_{0}\right)+\varphi^{\prime}(m) E_{\text {Independent }}\left[H u^{(0,1)}(X, H+\varphi(m) H)\right] \\
& +(\rho-1) p q h_{1} u^{(0,1)}\left(x_{1}, h_{1}+\varphi(m) h_{1}\right)-h_{2} u^{(0,1)}\left(x_{1}, h_{2}+\varphi(m) h_{2}\right) \\
& -h_{1} u^{(0,1)}\left(x_{2}, h_{1}+\varphi(m) h_{1}\right)+h_{2} u^{(0,1)}\left(x_{2}, h_{2}+\varphi(m) h_{2}\right)=0 .
\end{aligned}
$$

where

$$
\begin{aligned}
& \left.E_{\text {Independent }}\left[H u^{(0,1)}(X, H+\varphi(m) H)\right)\right]=p q h_{1} u^{(1,0)}\left(x_{1}, h_{1}+\varphi(m) h_{1}\right) \\
& \quad+p(1-q) h_{2} u^{(1,0)}\left(x_{1}, h_{2}+\varphi(m) h_{2}\right)+(1-p) q h_{1} u^{(1,0)}\left(x_{2}, h_{1}+\varphi(m) h_{1}\right) \\
& \quad+(1-p)(1-q) h_{2} u^{(1,0)}\left(x_{2}, h_{2}+\varphi(m) h_{2}\right) .
\end{aligned}
$$

Let

$$
\begin{gathered}
\Pi *=h_{1} u^{(0,1)}\left(x_{1}, h_{1}+\varphi(m) h_{1}\right)+h_{2} u^{(0,1)}\left(x_{2}, h_{2}+\varphi(m) h_{2}\right)-h_{2} u^{(0,1)} \\
\left(x_{1}, h_{2}+\varphi(m) h_{2}\right)-h_{1} u^{(0,1)}\left(x_{2} h_{1}+\varphi(m) h_{1}\right) .
\end{gathered}
$$

The first-order condition expressed at $m=m_{\perp}^{*}$ is non-negative if and only if $(\rho-1) \Pi *$ is non-negative. That is, $-h u^{(0,1)}(x, h)$ exhibits pair-wise risk aversion:

$$
\frac{\partial\left[-u^{(0,1)}(x, h)-h u^{(0,2)}(x, h)\right]}{\partial x} \leq 0 \Leftrightarrow-u^{(1,1)}(x, h)-h u^{(1,2)}(x, h) \leq 0 .
$$

if and only if $-h \frac{u^{(1,2)}(x, h)}{u^{(1,1)}(x, h)} \geq 1, \forall x, h$.

The first-order condition can be rewritten as follows:

$$
-u^{(1,0)}\left(y_{0}-m, h_{0}\right)+\varphi^{\prime}(m) E_{\text {Independent }}\left[H u^{(0,1)}(X, H+H \varphi(m))\right]+(\rho-1) p q \Pi *=0 .
$$

Assume that $X$ and $H$ are positively correlated $(\rho>1)$. Then, $m^{*} \geq m_{\perp}^{*}$ if the firstorder condition expressed with $m_{\perp}^{*}$ is non-positive:

$$
\begin{gathered}
\left.-u^{(1,0)}\left(y_{0}-m, h_{0}\right)+\varphi^{\prime}(m)\right) E_{\text {Independent }}\left[H u^{(0,1)}(X, H+H \varphi(m))\right]+(\rho-1) p q \Pi * \geq 0 . \\
\Leftrightarrow(\rho-1) p q \Pi * \geq 0 \\
\Leftrightarrow \Pi * \geq 0 .
\end{gathered}
$$

Thus the result follows. The positive correlation increases the value of the decision variable. For the case of negative correlation, the proof is the same.

The conditions in Proposition 3 are pair-wise risk-aversion coupled with relative risk aversion with respect to health greater than one. 
Proposition 5 bis: Assume the health production function is additive. If $u \in \mathcal{U}_{1,1-i c v}$ with $u^{(1,2)} \geq 0$, then

i. If $X$ and $H$ are positively correlated, then $m^{*} \geq m_{\perp}^{*}$.

ii. If $X$ and $H$ are negatively correlated, then $m^{*} \leq m_{\perp}^{*}$.

Proof The first-order condition is given by:

$$
\begin{gathered}
-u^{(1,0)}\left(y_{0}-m, h_{0}\right)+\Phi^{\prime}(m) \rho p q u^{(0,1)}\left(x_{1}, h_{1}+\Phi(m)\right)+p(1-\rho q) u^{(0,1)}\left(x_{1}, h_{2}+\Phi(m)\right) \\
+q(1-\rho p) u^{(0,1)}\left(x_{2}, h_{1}+\Phi(m)\right)+(1-p-q+\rho p q) u^{(0,1)}\left(x_{2}, h_{2}+\Phi(m)\right)=0 .
\end{gathered}
$$

Equivalently:

$$
\begin{gathered}
-u^{(1,0)}\left(y_{0}-m, h_{0}\right)+\Phi^{\prime}(m) E_{\text {Independent }}\left[u^{(0,1)}(X, H+\Phi(m))\right] \\
+(\rho-1) p q u^{(0,1)}\left(x_{1}, h_{1}+\Phi(m)\right)-u^{(0,1)}\left(x_{1}, h_{2}+\Phi(m)\right) \\
-u^{(0,1)}\left(x_{2}, h_{1}+\Phi(m)\right)+u^{(0,1)}\left(x_{2}, h_{2}+\Phi(m)\right)=0,
\end{gathered}
$$

where

$$
\begin{aligned}
& E_{\text {Independent }}\left[u^{(0,1)}(X, H+\Phi(m))\right]=p q u^{(1,0)}\left(x_{1}, h_{1}+\Phi(m)\right)+p(1-q) u^{(1,0)}\left(x_{1}, h_{2}+\Phi(m)\right) \\
& +(1-p) q u^{(1,0)}\left(x_{2}, h_{1}+\Phi(m)\right)+(1-p)(1-q) u^{(1,0)}\left(x_{2}, h_{2}+\Phi(m)\right) .
\end{aligned}
$$

Let $\Pi=u^{(0,1)}\left(x_{1}, h_{1}\right)+u^{(0,1)}\left(x_{2}, h_{2}\right)-u^{(0,1)}\left(x_{1}, h_{2}\right)-u^{(0,1)}\left(x_{2}, h_{1}\right)$,

$\Pi$ is non-negative (respectively non-positive) if and only if $u^{(0,1)}$ is super-modular (respectively sub-modular). That is, $u^{(1,2)} \geq 0$.

The first-order condition can be rewritten as follows:

$$
-u^{(1,0)}\left(y_{0}-m, h_{0}\right)+\Phi^{\prime}(m) E_{\text {Independent }}\left[u^{(0,1)}(X, H+m)\right]+(\rho-1) p q \Pi=0
$$

Assume that $X$ and $H$ are positively correlated $(\rho>1)$. Then, $m^{*} \geq m_{\perp}^{*}$ if the firstorder condition expressed with $m_{\perp}^{*}$ is non-positive:

$$
\begin{gathered}
-u^{(1,0)}\left(y_{0}-m, h_{0}\right)+\Phi^{\prime}(m) E_{\text {Independent }}\left[u^{(0,1)}(X, H+m)\right]+(\rho-1) p q \Pi \geq 0 \\
\Leftrightarrow(\rho-1) p q \Pi \geq 0 \\
\Leftrightarrow \Pi \geq 0 .
\end{gathered}
$$


Thus the result follows. The positive correlation increases the value of the decision variable. In case of negative correlation, the proof is the same.

In the case of additive health production function, the partial derivatives alternate in sign. The conditions are pair-wise risk aversion and cross-prudence with respect to health.

Conditions in Proposition 5 mean that the utility function belongs to $\mathcal{U}_{1,1-i c v}$ (the utility function exhibits pair-wise risk aversion) and the coefficient of relative risk aversion of $u^{(1,0)}$ is greater than one. The last condition implies $u^{(1,2)} \geq 0$, meaning that the utility function exhibits cross-prudence with respect to health. Therefore, conditions in Proposition 5 are more stringent than those in Proposition 5 bis.

\subsection{Increasing the Correlation}

Epstein and Tanny (1980) show that an increase in the dependence on parameter $\rho$ is disliked by any bivariate risk-averse decision-maker $\left(u^{(1,1)} \leq 0\right.$, ). That is,

$$
\rho_{1} \geq \rho_{2} \Rightarrow\left(X_{1}, H_{1}\right) \preccurlyeq_{1,1-i c v}\left(X_{2}, H_{2}\right),
$$

where $\rho_{1}$ and $\rho_{2}$ are the respective dependence parameters of $\left(X_{1}, H_{1}\right)$ and $\left(X_{2}, H_{2}\right)$. Let $m_{1}^{*}$ and $m_{2}^{*}$ denote the respective demand for health care.

Intuitively, the more health risk and the wealth risk are correlated, the larger the demand for health care. This is true for pair-wise risk-averse decision-makers because they dislike an increase in the correlation (see Epstein and Tanny 1980). We have the following result:

Proposition 6 Assume the health production function is multiplicative. If $u \in \mathcal{U}_{1,1-i c v}$ with $-h \frac{u^{(0,2)}(c, h)}{u^{(0,1)}(c, h)} \geq 1 ;-h \frac{u^{(1,2)}(c, h)}{u^{(1,1)}(c, h)} \geq 1, \forall c, h$, then $\rho_{1} \geq \rho_{2} \Rightarrow m_{1}^{*} \geq m_{2}^{*}$.

Proof $\rho_{1} \geq \rho_{2} \Rightarrow\left(X_{1}, H_{1}\right) \preccurlyeq_{1,1-i c v}\left(X_{2}, H_{2}\right)$.

Thus applying Theorem 1 gives the result.

Proposition 6 bis Assume the health production function is additive. If $u \in \mathcal{U}_{1,2-i c v}$ then, $\rho_{1} \geq \rho_{2} \Rightarrow m_{1}^{*} \geq m_{2}^{*}$.

\section{Proof}

$$
\rho_{1} \geq \rho_{2} \Rightarrow\left(X_{1}, H_{1}\right) \preccurlyeq_{1,1-i c v}\left(X_{2}, H_{2}\right) .
$$

Thus applying Theorem 2 gives the result.

Increasing the dependence increases the optimal value of the amount invested in health care when the decision-maker exhibits pair-wise risk aversion. From a policy perspective this proposition suggests that policy measures which increase 
the correlation between health and wealth will tend to result in an increase in the demand for health care.

\subsection{Quadrant Dependence}

Let us deal with a more general concept of dependence, namely that of quadrant dependence. We assume that $(X, H)$ is positively (negatively respectively) quadrant dependent. Recall the definition of quadrant dependence:

\section{Definition 6 Lehman (1966)}

i. $(X, H)$ is said to be positively quadrant dependent if

$$
P(X>x, H>h) \geq P(X>x) P(H>h), \forall x, h .
$$

ii. $(X, H)$ is said to be negatively quadrant dependent if

$$
P(X>x, H>h) \leq P(X>x) P(H>h), \forall x, h .
$$

Let us define the bivariate $\operatorname{risk}\left(X^{\perp}, H^{\perp}\right)$ such that:

- $X^{\perp}$ and $X$ have the same distribution,

- $H^{\perp}$ and $H$ have the same distribution and,

- $X^{\perp}$ and $H^{\perp}$ are independent.

We want to compare the optimal amount obtained with the dependence between the two sources of risks $\left(m^{*}\right)$ with that obtained in the case of independence $\left(m_{\perp}^{*}\right)$. Denuit et al. (1999a, b) show that:

i. $(X, H)$ positively quadrant dependent is equivalent to $(X, H) \preccurlyeq_{1,1_{-i c y}}\left(X^{\perp}, H^{\perp}\right)$;

ii. $(X, Y)$ negatively quadrant dependent is equivalent to $\left(X^{\perp}, H^{\perp}\right) \preccurlyeq_{1,1_{\text {-icv }}}(X, H)$.

Let $m_{\perp}^{*}$ be the optimal decision in the case of independence. Intuition suggests that positive quadrant dependence will increase the optimal amount invested in health care compared to independence, whereas negative quadrant dependence will decrease it. We have the following propositions:

Proposition 7 Assume the health production function is multiplicative. If $u \in \mathcal{U}_{1,1-i c v}$ with

$$
-h \frac{u^{(0,2)}(c, h)}{u^{(0,1)}(c, h)} \geq 1 ;-h \frac{u^{(1,2)}(c, h)}{u^{(1,1)}(c, h)} \geq 1, \quad \forall c, h .
$$

i. If $(X, H)$ is positively quadrant dependent, then $m^{*} \geq m_{\perp}^{*}$.

ii. If $(X, H)$ is negatively quadrant dependent, then $m^{*} \leq m_{\perp}^{*}$. 
Proof The proof is direct by applying Theorem 1 and the fact that:

i. $(X, H)$ positively quadrant dependent is equivalent to $(X, H) \preccurlyeq_{1,1_{-i c v}}\left(X^{\perp}, H^{\perp}\right)$.

ii. $(X, H)$ negatively quadrant dependent is equivalent to $\left(X^{\perp}, H^{\perp}\right) \aleph_{1,1_{-i c v}}(X, H)$.

Proposition 7 bis Assume the health production function is additive. If $u \in \mathcal{U}_{1,2-i c v}$

i. If $(X, H)$ is positively quadrant dependent, then $m^{*} \geq m_{\perp}^{*}$.

ii. If $(X, H)$ is negatively quadrant dependent, then $m^{*} \leq m_{\perp}^{*}$.

Proof The proof is direct by applying Theorem 2 and the fact that:

i. $(X, H)$ positively quadrant dependent is equivalent to $(X, H) \preccurlyeq_{1,1_{-i c v}}\left(X^{\perp}, H^{\perp}\right)$.

ii. $(X, H)$ negatively quadrant dependent is equivalent to $\left(X^{\perp}, H^{\perp}\right) \aleph_{1,1_{-i c v}}(X, H)$.

That is, when the wealth risk and the health risk are dependent via the concept of positive quadrant dependence, the decision-maker chooses an optimal value of the decision variable higher than what he or she could choose in the event of independence. Negative dependence reverses the inequality because poor health is for the most part offset by large wealth and low wealth is offset by good health. This is a form of homemade diversification.

\section{Effectiveness of Medical Treatments}

The decision maker sacrifices some consumption in order to improve future health status via the health production function. However with uncertainty surrounding the effectiveness of future medical care, the final health status taking into account the health production function and the uncertainty will be as follows: $\psi(m, H)+\in$ with $E(\in)=0$ where $\in$ determines the efficiency of medical treatment in the future. We assume that $\in$ and $H$ are independent. Thus, we face a Rothschild and Stiglitz (1970) increase in risk. The decision-maker maximises:

$$
u\left(y_{0}-m, h_{0}\right)+E u(X, \psi(m, H)+\in) .
$$

Assume that all the risks are independent and define the derived utility function as follows:

$$
V(w, h)=E u(w, h+\in) .
$$

Therefore, the decision-maker maximises:

$$
u\left(y_{0}-m, h_{0}\right)+E V(X, \psi(m, H)) .
$$


Let $m_{\in}^{*}$, denote the optimal expenses in health care in presence of $\in$. We want to point out conditions under which $m_{\in}^{*}$ is greater than $m_{0}^{*}$, the optimal expenses in health care without $\in$. We have the following result:

Proposition 8 If $u \in \mathcal{U}_{1,2-i c v}$, then $m_{\in}^{*} \geq m^{*}$.

Proof The proof is direct by applying Theorem 2 and the fact that $(X, H+\in) \preccurlyeq_{1,2_{-i c v}}(X, H)$.

This result is intuitive because the uncertainty surrounding the efficiency of the treatments plays the role of white noise. Therefore, the situation in presence of this uncertainty is like the initial one in presence of the health and wealth risks but with more global risk. The decision-maker must be risk-averse with respect to health and pair-wise risk, while being cross-prudent with respect to health to at least guarantee an increase in the demand for health care, regardless of the form of the health production function. We can notice that the comparison between $m_{\in}^{*}$ and $m_{0}^{*}$ corresponds to analysing the conditions in which the decision-maker with utility function $V$ will demand more health care than the decision-maker with utility function $u$ in presence of wealth and health risks.

\section{Conclusion}

In this paper, we analyse the behaviour of a decision-maker with a multiplicative/ additive health production function with a bivariate utility function facing simultaneous health and wealth risk (the bivariate risk). We study the general changes in the bivariate risk caused by the $\left(s_{1}, s_{2}\right)$ - increasing concave order and show that the optimal value of the amount invested in health care decreases when the utility function belongs to the class of $\left(s_{1}, s_{2}\right)$ - increasing concave functions and the coefficients of generalised partial risk aversion of order $\left(s_{1}, s_{2}\right)$ are less than $k_{1}$.

In increasing health risk (endogenous risk) or wealth risk (exogenous risk) induces an increase in the demand for health care and causes the decision-maker to build health capital. We generalise the results concerning the effects of wealth risk acting as a background risk on the optimal amount invested in health care. We conclude that increasing correlation causes an increase in the optimal investment. Positive dependence increases the optimal value of the choice variable whereas negative dependence decreases it. With uncertainty surrounding the effectiveness of future medical care, the demand for health care also increases as with the initial case. The conditions are more stringent with a multiplicative health production function than with additive health production function.

In terms of future work, we would like to investigate extensions to our current work. In particular, alternative methods of modelling dependencies between risks could be investigated. For instance the application of copulas for modelling dependencies between risk factors was considered a favourable method (prior to the Global Financial Crisis), its application in health and wealth risk settings may be more 
viable. We would also like to investigate alternative method of risk management to determine the optimal outcomes for decision makers. This is particularly pertinent given that risk management is an active area of research where innovations are frequently occurring.

Open Access This article is distributed under the terms of the Creative Commons Attribution 4.0 International License (http://creativecommons.org/licenses/by/4.0/), which permits unrestricted use, distribution, and reproduction in any medium, provided you give appropriate credit to the original author(s) and the source, provide a link to the Creative Commons license, and indicate if changes were made.

\section{References}

Ahangar, A., Ahmadi, A. M., Mozayani, A. H., \& Dizaji, S. F. (2018). Why are risk-pooling and risksharing arrangements necessary for financing healthcare and improving health outcomes in low and lower middle-income countries. Health, 10(01), 122-131.

Chang, F. (1996). Uncertainty and investment in health. Journal of Health Economics, 15, 369-376.

Cropper, M. (1977). Health, investment in health and occupational choice. Journal of Political Economy, 85, 1273-1294.

Dardanoni, V., \& Wagstaff, A. (1987). Uncertainty, inequalities in health and the demand for health. Journal of Health Economics, 6, 283-290.

Dardanoni, V., \& Wagstaff, A. (1990). Uncertainty and the demand for medical care. Journal of Health Economics, 9, 23-38.

Denuit, M., Eeckhoudt, L., \& Menegatti, M. (2009). Correlated risks, bivariate utility and optimal choices. Economic Theory, 46(1), 39-54.

Denuit, M., Lefevfre, C., \& Mesfiou, M. (1999a). A class of stochastic ordering, with applications in actuarial sciences. Insurance: Mathematics and Economics, 24, 31-50.

Denuit, M., Lefevfre, C., \& Mesfiou, M. (1999b). Stochastic ordering of convex-type for discrete bivariate risks. Scandinavian Actuarial Journal, 1, 32-51.

Drèze, J., \& Modigliani, M. (1972). Consumption decision under uncertainty. Journal of Economic Theory, 5, 308-335.

Edwards, R. D. (2008). Health risk and portfolio choice. Journal of Business and Economic Statistics, 26, $472-485$.

Eeckhoudt, L., Rey, B., \& Schlesinger, H. (2007). A good sign for multivariate risk taking. Management Science, 53, 117-124.

Eeckhoudt, L., \& Schlesinger, H. (2008). Changes in risk and the demand for savings. Journal of Monetary Economics, 55, 1329-1336.

Epstein, L. G., \& Tanny, S. M. (1980). Increasing generalized correlation: A definition and some economic consequences. Canadian Journal of Economics, Canadian Economics Association, 13(1), 16-34.

Fan, E., \& Zhao, R. (2009). Health status and portfolio choice: Causality or heterogeneity? Journal of Banking \& Finance, 33, 1079-1088.

Finkelstein, A., Hendren, N., \& Shepard, M. (2019). Subsidizing health insurance for low-income adults: Evidence from Massachusetts. American Economic Review, 109(4), 1530-1567.

Gollier, C., \& Pratt, J. (1996). Risk vulnerability and the tempering effect of background risk. Econometrica, 64, 1109-1123.

Grossman, M. (1972). On the concept of health and the demand for health. Journal of Political Economy, $80,223-255$.

Heaton, J., \& Lucas, D. (2000). Portfolio choice and asset prices: The importance of entrepreneurial risk. Journal of Finance, 55, 1163-1198.

Jokung, O. (2011). Bivariate risk apportionment via stochastic dominance. Journal of Mathematical Economics, 47, 448-452.

Keenan, D., Rudow, D., \& Snow, A. (2008). Risk preferences and changes in background risk. Journal of Risk and Uncertainty, 36, 139-152.

Kimball, M. (1990). Precautionary saving in the small and in the large. Econometrica, 58, 53-73. 
Kimball, M. (1993). Standard risk aversion. Econometrica, 61, 589-611.

Kimball, M., \& Weil, P. (2009). Precautionary saving and consumption smoothing across time and possibilities. Journal of Money, Credit \& Banking, 41, 245-284.

Lajeri-Chaherli, F. (2004). Proper prudence, standard prudence and precautionary vulnerability. Economics Letters, 82, 29-34.

Lehmann, E. (1966). Some concepts of dependence. Annals of Mathematics and Statistics, 37, $1137-1153$

Leland, H. (1968). Saving and uncertainty: the precautionary demand for saving. Quarterly Journal of Economics, 82, 465-473.

Markowitz, H. (1952). Portfolio selection. The Journal of Finance, 7(1), 77-91.

Meer, J., Miller, D., \& Rosen, H. S. (2003). Exploring the health-wealth nexus. Journal of Health Economics, 22, 713-730.

Palumbo, M. G. (1999). Uncertain medical expenses and precautionary saving near the end of the lifecycle. Review of Economic Studies, 66, 395-421.

Pang, G., \& Warshawsky, M. (2010). Optimizing the equity-bond-annuity portfolio in retirement: The impact of uncertain health expenses. Insurance: Mathematics and Economics, 46, 198-209.

Pratt, J. W. (1964). Risk aversion in the small and in the large. Econometrica, 32(1-2), 122-136.

Pratt, J., \& Zeckhauser, R. (1987). Proper risk aversion. Econometrica, 55, 143-154.

Richard, S. F. (1975). Multivariate risk aversion, utility independence and separable utility functions. Management Science, 22, 12-21.

Rosen, H. S., \& Wu, S. (2004). Portfolio choice and health status. Journal of Financial Economics, 72, 457-484.

Rothschild, M., \& Stiglitz, J. (1970). Increasing risk: I. A definition. Journal of Economic Theory, 2, 225-243.

Sandmo, A. (1970). The effect of uncertainty on saving decision. Review of Economics Studies, 37, 353-360.

Scarsini, M. (1985). Stochastic dominance with pair-wise risk aversion. Journal of Mathematical Economics, $14,187-201$.

Selden, T. (1993). Uncertainty and health care spending by poor: The health capital model revisited. Journal of Health Economics, 12, 109-115.

Wang, J., \& Li, J. (2010). Multiplicative risk apportionment. Mathematical Social Science, 60, 79-81.

Publisher's Note Springer Nature remains neutral with regard to jurisdictional claims in published maps and institutional affiliations. 